\title{
Genuine photon-magnon-phonon Einstein-Podolsky-Rosen steerable nonlocality in a continuously-monitored cavity magnomechanical system
}

\author{
Huatang Tan \\ Department of Physics, Huazhong Normal University, Wuhan 430079, China
}

(Received 28 August 2019; published 9 December 2019)

\begin{abstract}
In this paper, we propose a scheme for generating genuine tripartite steering nonlocality in a cavity magnomechanical system composed of an yttrium iron garnet (YIG) sphere with a diameter of a few hundred micrometers inside a microwave cavity. In the system, the magnons, i.e., collective spin excitations in the sphere, are coupled to the cavity photons via magnetic-dipole interaction and at the same time coupled to phonons, the quanta of vibration of the sphere, by magnetostrictive interaction. We consider that the output field of the driven microwave cavity is subject to a time-continuous homodyne detection. We find that without the continuous measurement, only weak bipartite steering among the photons, magnons, and phonons can be obtained and the genuine tripartite steering is unachievable, although there exists weak genuine tripartite entanglement; when the continuous measurement is present, the bipartite steering is enhanced considerably and furthermore the genuine photon-magnon-phonon tripartite steering can be generated in the steady-state regime. It is shown that the generated tripartite steering is robust against thermal fluctuations for realistic parameters. Our scheme opens a promising route for exploring and exploiting macroscopic quantum effects in such a macroscopic quantum interface of photons, magnons, and phonons.
\end{abstract}

DOI: 10.1103/PhysRevResearch.1.033161

\section{INTRODUCTION}

Recently, hybrid systems based on magnons - quanta of spin waves in magnetic materials-have attracted considerable attention, due to great frequency tunability and very low loss of magnons [1]. Experiments have realized strong coherent coupling of magnons to cavity photons [2-5] and related phenomena, such as the exceptional point [6], bistability of cavity magnon polaritons [7], and optical cooling of magnons [8]. Besides, resolving magnon Fock states by coupling of magnons in a YIG sphere to a superconducting quantum qubit has been achieved [9-11]. Further, the simultaneous coupling of magnons in a YIG sphere to cavity photons and phonons due to the sphere's vibration has been demonstrated [12]. Cavity magnomechanical systems, in parallel with cavity optomechanical systems [13], represent a promising platform for studying macroscopic quantum phenomena [14,15], since the YIG sphere is around hundreds of micrometers in diameter, at the macroscopic scale. More interestingly, recent studies have already shown that quantum effects, such as quadrature squeezing, entanglement, and magnon quantum blockade, can be generated in cavity magnomechanics [16-19]. These findings inspire us to further explore novel quantum phenomena in the hybrid macroscopic quantum interface of photons, magnons, and phonons.

Published by the American Physical Society under the terms of the Creative Commons Attribution 4.0 International license. Further distribution of this work must maintain attribution to the author(s) and the published article's title, journal citation, and DOI.
Einstein-Podolsky-Rosen (EPR) steering [20,21], originally termed by Schödinger in his response to the famous EPR paradox [22], characterizes one's capability to control the states of a remote particle via local measurements on his/her particle entangled with the remote one. EPR steering is an intrinsic quantum nonlocal effect and it is intermediate between Bell nonlocality [23] and entanglement [24,25]. Moreover, distinct from Bell nonlocality and entanglement, EPR steering is essentially asymmetric with respect to two observers [26-28]. Besides being of fundamental interest, EPR steering has potential applications, e.g., one-sided device-independent quantum cryptography $[29,30]$, subchannel discrimination [31], and secure quantum teleportation [32]. Currently, EPR steering has already been realized experimentally in a variety of systems [26-28,33-36].

Here, we consider the generation of genuine photonmagnon-phonon EPR steering nonlocality by cavity magnomechanics. In this system, the magnons are simultaneously coupled to the cavity photons via magnetic-dipole interaction and to phonons of the vibrational sphere by magnetostrictive interaction. We consider that the cavity is subject to timecontinuous homodyne detection which has frequently been employed to control cavity optomechanical systems [37-39]. We find that in the absence of the continuous measurement, only weak bipartite steering among the photons, magnons, and phonons can be achieved. When the measurement is present, the bipartite steering is enhanced considerably and strong genuine photon-magnon-phonon tripartite steering can be generated in the steady-state regime.

The remainder of this paper is organized as follows. In Sec. II, the system is introduced and the working equations are presented. In Sec. III, the quantum steering properties of 


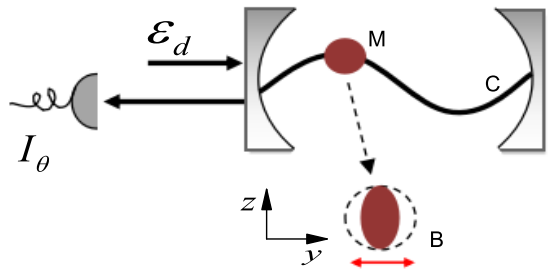

FIG. 1. The schematic plot of a cavity magnomechanical system, where a YIG sphere in a uniform bias magnetic field along $z$ direction is placed at the maximum of the electromagnetic field inside a driven microwave cavity. The magnon mode ( $\hat{M}$, spin waves in the sphere) is coupled to the cavity mode $(\hat{C})$ via magnetic dipole interaction and also coupled to the phonon mode ( $\hat{B}$, vibration of the sphere) by magnetostrictive interaction. The cavity output is subject to a timecontinuous homodyne detection, with the detection current $I_{\theta}$.

the continuously-monitored cavity magnomechanical system are investigated in detail. In the last Sec. IV, we give the main summary.

\section{MODEL}

As schematically shown in Fig. 1, we consider a cavity magnomechanical system which has already been experimentally realized very recently [12]. In the system, a $250-\mu \mathrm{m}-$ diameter YIG sphere is placed at the maximum of electromagnetic field inside a microwave cavity which is strongly pumped by an external microwave source. The YIG sphere is also in a uniform magnetic field biased along the $z$ direction. The magnons, which characterize the collective excitations of the spin ensemble in the YIG sphere in the external uniform magnetic field, are coupled to the cavity photons via magnetic dipole interaction and at the same time are also coupled to phonons from the vibration of deformation of the sphere via the magnetostrictive coupling. In the rotating frame at the frequency $\omega_{d}$ of the driving field, the Hamiltonian of the system reads $(\hbar=1)$

$$
\begin{aligned}
\hat{H}= & \delta_{c} C^{\dagger} \hat{C}+\delta_{m} \hat{M}^{\dagger} \hat{M}+\omega_{b} \hat{B}^{\dagger} \hat{B}+g_{c m}\left(\hat{C}^{\dagger} \hat{M}+\hat{C} \hat{M}^{\dagger}\right) \\
& +g_{b m} \hat{M}^{\dagger} \hat{M}\left(\hat{B}+\hat{B}^{\dagger}\right)-i\left(\mathcal{E}_{d}^{*} \hat{C}-\mathcal{E}_{d} \hat{C}^{\dagger}\right),
\end{aligned}
$$

where the bosonic annihilation (creation) operators $\hat{C}\left(\hat{C}^{\dagger}\right)$, $\hat{M}\left(\hat{M}^{\dagger}\right)$, and $\hat{B}\left(\hat{B}^{\dagger}\right)$ denote, respectively, the cavity photon, magnon, and phonon modes whose resonances are taken to be $\omega_{c}, \omega_{m}$, and $\omega_{b}$. The detuning $\delta_{c}=\omega_{c}-\omega_{d}$ and $\delta_{m}=\omega_{m}-$ $\omega_{d}$. The magnon frequency $\omega_{m}=\gamma H$, where $\gamma$ is the gyromagnetic ratio and $H$ is the magnetic field strength. The parameters $g_{c m}$ and $g_{b m}$, respectively, represent the optomagnon and magnomechanical coupling strengths, and $\left|\mathcal{E}_{d}\right| \equiv \sqrt{\frac{\kappa_{c} P_{\mathrm{w}}}{\hbar \omega_{c}}}$ denotes the amplitude of the driving field with the power $P_{\mathrm{w}}$. Experimentally, the magnon-photon coupling $g_{c m}$ can be larger than the dissipation rates $\kappa_{c}$ and $\kappa_{m}$ of the cavity and magnon linewidths $[2,3]$, while the magnon-phonon coupling $g_{b m}$ is typically weak but it can be effectively enhanced with strong driving field.

For strong driving, Eq. (1) can be linearized around the steady-state amplitudes by expressing the operator $\hat{J}(J=$ $\{C, M, B\})$ as $\hat{J}=\langle\hat{J}\rangle_{\mathrm{ss}}+\hat{j}(j=\{c, m, b\})$, provided that $\left|\langle\hat{J}\rangle_{\mathrm{ss}}\right|^{2} \gg\left\langle\hat{j}^{\dagger} \hat{j}\right\rangle$, where $\langle\hat{J}\rangle_{\mathrm{ss}}$ and the operators $\hat{j}$ respectively represent the steady-state amplitudes and quantum fluctuations of the corresponding modes. We have the steady-state amplitudes

$$
\langle\hat{M}\rangle_{\mathrm{ss}}=\frac{-4 i g_{c m} \mathcal{E}_{d}}{\left(\kappa_{m}+2 i \Delta_{m}\right)\left(\kappa_{c}+2 i \delta_{c}\right)+4 g_{c m}^{2}},
$$

$\langle\hat{C}\rangle_{\mathrm{ss}}=\frac{\left(2 \Delta_{m}-i \kappa_{m}\right)\langle\hat{M}\rangle_{\mathrm{ss}}}{2 g_{c m}}$, and $\langle\hat{B}\rangle_{\mathrm{ss}}=-\frac{2 g_{b m}\left|\langle\hat{M}\rangle_{\mathrm{ss}}\right|^{2}}{2 \omega_{b}-i \kappa_{b}}$, where the effective detuning $\Delta_{m}=\delta_{m}+2 g_{b m} \operatorname{Re}\left[\langle\hat{B}\rangle_{\text {ss }}\right]$ and $\kappa_{b}$ the damping rate of the phonon mode. The linearized Hamiltonian can be derived as

$$
\begin{aligned}
\hat{H}_{\text {lin }}= & \delta_{c} \hat{c}^{\dagger} \hat{c}+\Delta_{m} \hat{m}^{\dagger} \hat{m}+\omega_{b} \hat{b}^{\dagger} \hat{b}+g_{c m}\left(\hat{c}^{\dagger} \hat{m}+\hat{m}^{\dagger} \hat{c}\right) \\
& +G_{b m}\left(\hat{m}+\hat{m}^{\dagger}\right)\left(\hat{b}+\hat{b}^{\dagger}\right),
\end{aligned}
$$

where the enhanced magnon-phonon coupling $G_{b m} \equiv$ $\langle\hat{M}\rangle_{\mathrm{ss}} g_{b m}$ (taken to be real). We see that the cavity and magnon modes couple to each other via a beam-splitter-like linear mixing, while the magnon and phonon modes form quantumnondemolition-measurement-like coupling which can generate nonclassical states, as we will show later.

We further consider that the cavity output field $\hat{c}_{\text {out }}(t)$ is subject to time-continuous homodyne detection. For the cavity output $\hat{c}_{\text {out }}(t)=\sqrt{\kappa_{c}} \hat{c}+\hat{c}_{\text {in }}(t)$, where $\hat{c}_{\text {in }}(t)$ is the input vacuum of the cavity satisfying the nonzero correlations $\left\langle\hat{c}_{\text {in }}(t) \hat{c}_{\text {in }}^{\dagger}\left(t^{\prime}\right)\right\rangle=\delta\left(t-t^{\prime}\right)$, the generalized quadrature

$$
\hat{x}_{\text {out }}^{\theta}=\frac{1}{\sqrt{2}}\left(\hat{c}_{\text {out }} e^{-i \theta}+\hat{c}_{\text {out }}^{\dagger} e^{i \theta}\right)
$$

is continuously homodyned, with $\theta$ the phase of the local field. The detection current

$$
I_{\theta} d t=\sqrt{\kappa_{c} \eta}\left\langle\hat{c} e^{-i \theta}+\hat{c}^{\dagger} e^{i \theta}\right\rangle d t+d W,
$$

where $\eta$ accounts for the detection efficiency and $d W$ is the Wiener increment which satisfies $(d W)^{2}=d t$. Conditioned on the detection result, the stochastic master equation for the conditional state $\hat{\rho}_{s}^{c}$ of the whole system is given by $[40,41]$

$$
\begin{aligned}
d \hat{\rho}_{s}^{c}= & -i\left[\hat{H}_{\text {lin }}, \hat{\rho}_{s}^{c}\right] d t+\sum_{o=m, c} \kappa_{o} \mathcal{L}[\hat{o}] \hat{\rho}_{s}^{c} d t \\
& +\kappa_{b}\left(\bar{n}_{b}^{\mathrm{th}}+1\right) \mathcal{L}[\hat{b}] \hat{\rho}_{s}^{c} d t+\kappa_{b} \bar{n}_{b}^{\mathrm{th}} \mathcal{L}\left[\hat{b}^{\dagger}\right] \hat{\rho}_{s}^{c} d t \\
& +\sqrt{\kappa_{c} \eta}\left[(\hat{c}-\langle\hat{c}\rangle) \hat{\rho}_{s}^{c} e^{-i \theta}+\text { H.c. }\right] d W,
\end{aligned}
$$

where the symbol $\mathcal{L}[\hat{o}] \hat{\rho}=\hat{o} \hat{\rho} \hat{o}^{\dagger}-\frac{1}{2}\left(\hat{o}^{\dagger} \hat{o} \hat{\rho}+\hat{\rho} \hat{o}^{\dagger} \hat{o}\right)$, the second terms in the first line describe the dissipation of the cavity and magnon modes into vacuum, the second line describes the damping of the phonon mode in Markovian thermal environment with the mean thermal excitation number $\bar{n}_{b}^{\text {th }} \equiv$ $\left(e^{\hbar \omega_{b} / k_{B} T}-1\right)^{-1}$ at temperature $T, k_{B}$ is the Boltzmann constant, and the last line characterizes the measurement-induced backaction effect.

When the system starts from a Gaussian state, determined by Eq. (6), it evolves in Gaussian states which are completely determined by the correlation matrix $\sigma_{c, j j^{\prime}}=\left\langle\mu_{j} \mu_{j^{\prime}}+\right.$ $\left.\mu_{j^{\prime}} \mu_{j}\right\rangle / 2-\left\langle\mu_{j}\right\rangle\left\langle\mu_{j^{\prime}}\right\rangle$, where $\mu=\left(\hat{X}_{c}, \hat{Y}_{c}, \hat{X}_{m}, \hat{Y}_{m}, \hat{X}_{b}, \hat{Y}_{b}\right)$ and the quadrature operators $\hat{X}_{o}=\left(\hat{o}+\hat{o}^{\dagger}\right) / \sqrt{2}$ and $\hat{Y}_{o}=-i(\hat{o}-$ $\left.\hat{o}^{\dagger}\right) / \sqrt{2}$. From Eq. (6), the first moments $\left\langle\mu_{j}\right\rangle$ and the 
correlation matrix $\sigma$ are governed by

$$
\begin{aligned}
\frac{d}{d t}\left\langle\mu^{T}\right\rangle & =A\left\langle\mu^{T}\right\rangle+\left(\sigma_{c} C-\Gamma\right) d W \\
\frac{d}{d t} \sigma_{c} & =A \sigma_{c}+\sigma_{c} A^{T}+D-\left(\sigma_{c} C-\Gamma\right)\left(\sigma_{c} C-\Gamma\right)^{T} .
\end{aligned}
$$

Here, the drift matrix

$$
A=\left(\begin{array}{ccc}
A_{c} & A_{c m} & 0 \\
A_{c m} & A_{m} & A_{b m} \\
0 & A_{b m} & A_{b}
\end{array}\right),
$$

where $A_{o}=-\frac{1}{2}\left(\begin{array}{cc}\kappa_{o} & -2 \Delta_{o} \\ 2 \Delta_{o} & \kappa_{o}\end{array}\right)$, with $\Delta_{c}=\delta_{c}$ and $\Delta_{b}=\omega_{b}$, $A_{c m}=\left(\begin{array}{cc}0 & g_{c m} \\ -g_{c m} & 0\end{array}\right)$, and $A_{b m}=-\left(\begin{array}{cc}0 & 0 \\ 2 G_{b m} & 0\end{array}\right)$. The matrix $D=$ $\operatorname{diag}\left(D_{c}, D_{m}, D_{b}\right)$, where $D_{c, m}=\frac{\kappa_{c, m}}{2} I_{2}$ and $D_{b}=\kappa_{b}\left(\bar{n}_{b}^{\text {th }}+\right.$ $\left.\frac{1}{2}\right) I_{2}$, with $I_{2}$ the $2 \times 2$ identity matrix. The vector $C$ describes the homodyne detection and $\Gamma$ is related to the system's noise prosperities (the correlations between the cavity input noise and the detection noise), given by

$$
\begin{aligned}
C^{T} & =\sqrt{2 \kappa_{c} \eta}(\cos \theta,-\sin \theta, 0,0,0,0), \\
\Gamma^{T} & =\sqrt{\frac{\kappa_{c} \eta}{2}}(\cos \theta,-\sin \theta, 0,0,0,0) .
\end{aligned}
$$

It is shown from Eqs. (7a) and (7b) that the first moments are stochastic and they are dependent on the detection results, while the correlation matrix $\sigma_{c}$ is deterministic and independent of the detection outcomes. Thus, in phase space the first moments undergo random walk and the deterministic variances for each mode's center at the trajectory of the first moments [quantum trajectories of Eq. (6)]. To convert the conditional correlation matrix into the unconditional one, we can introduce an active feedback, with the strength appropriate to the detection outcomes, to drive the system (displacement operation) and remove the first moments [42]. In addition, it is shown from Eq. (7b) that the time-continuous measurements not only modify the drift and diffusion matrices of the system, but also induce nonlinear terms (last term) due to the measurement-induced backaction. In the absence of the measurement $\eta=0$, Eq. (13a) for the first-order terms disappears and the nonlinear equation of Eq. (13b) reduces to the linear one with drift and diffusion matrices $A$ and $D$.

The stability of the system without the detection is that all eigenvalues of the drift matrix $A$ have negative real parts, while with the detection the stable condition is [40]

$$
C x_{\lambda} \neq 0 \forall x_{\lambda}: \tilde{A} x_{\lambda}=\lambda x_{\lambda},
$$

with $\operatorname{Re}(\lambda) \geqslant 0$ and the new $\operatorname{drift} \tilde{A}=A+\Gamma C^{T}$. The stability condition means that whenever all the degrees of freedom that are not strictly stable under the drift matrix $\tilde{A}$ contribute to the measurement output related to $C$. Therefore, even if the unconditional correlation matrix, in the absence of the measurement, is instable, the conditional correlation matrix determined by Eq. (7b) can still be stable.

\section{RESULTS}

We now discuss the quantum steerable correlations in the present system. The two-mode steering from mode $j$ to mode $i(i \neq j) \in\{c, m, b\}$ can be verified by the following inequality [43]:

$$
S_{i \mid j}=V_{\text {inf }}\left(\hat{X}_{i}^{\theta_{i}}\right) V_{\text {inf }}\left(\hat{Y}_{i}^{\theta_{i}}\right)<1 / 4,
$$

where the inferred variances $V_{\text {inf }}\left(\hat{X}_{i}^{\theta_{i}}\right)=V\left(\hat{X}_{i}^{\theta_{i}}+f_{j x} X_{j}^{\theta_{j}}\right)$ and $V_{\text {inf }}\left(\hat{Y}_{i}^{\theta_{i}}\right)=V\left(\hat{Y}_{i}^{\theta_{i}}+f_{j y} Y_{j}^{\theta_{j}}\right)$, conditioned on the results of the local measurements on the $j$ mode. The gain factors $f_{j x(y)}$ and $f_{j y}$ and the angles $\theta^{i, j}$ are chosen such that the inferred variances minimize. They can be determined in the optical verification of the steering via transferring the magnonic and phononic states to optical modes which are subjected to homodyne detection (which will be discussed later). The steering parameter $S_{i \mid j} \rightarrow 0$ means a nearly perfect EPR paradox and the smaller the quantity $S_{i \mid j}$ shows stronger two-mode steering. Based on the bipartite steering, the genuine tripartite steering among the cavity, magnon, and phonon modes can be necessarily verified by the inequality [44]

$$
S_{c m b}=\left(S_{c \mid m b}+S_{m \mid c b}+S_{b \mid c m}\right)<1 / 4,
$$

where the parameters $S_{i \mid j k} \equiv V_{\text {inf }}\left(\hat{X}_{i}^{\theta_{i}}\right) V_{\text {inf }}\left(\hat{Y}_{i}^{\theta_{i}}\right)(i \neq j \neq k \in$ $\{c, m, b\})$ quantify the steering between mode $i$ and the subgroup of the remaining $j$ and $k$ modes. Similarly, the inferred variances $V_{\text {inf }}\left(\hat{X}_{i}^{\theta_{i}}\right)=V\left(\hat{X}_{i}^{\theta_{i}}+f_{j x} X_{j}^{\theta_{j}}+f_{k x} X_{k}^{\theta_{k}}\right)$ and $V_{\text {inf }}\left(\hat{Y}_{i}^{\theta_{i}}\right)=V\left(\hat{Y}_{i}^{\theta_{i}}+f_{j y} Y_{j}^{\theta_{j}}+f_{k y} Y_{k}^{\theta_{k}}\right)$. It is obvious that when Eq. (12) is held, it means that the bipartite steering between the arbitrary $i$ mode and the party of the remaining two modes is achievable.

In Fig. 2, the steady-state steering quantities $S_{i \mid j k}$ and $S_{c m b}$ versus the detunings $\Delta_{m}$ and $\delta_{a}$ are plotted for the cases without and with the continuous measurement. The parameters are close to those utilized in a recent experiment in Ref. [12], as the phonon frequency $\omega_{b} / 2 \pi=10 \mathrm{MHz}$, the cavity dissipation rate $\kappa_{c} / 2 \pi=3 \mathrm{MHz}$, the magnon loss rate $\kappa_{m} / 2 \pi=$ $1 \mathrm{MHz}$, the phonon damping rate $\kappa_{b} / 2 \pi=300 \mathrm{~Hz}$, the coupling $g_{c m}=G_{b m}=5 \mathrm{MHz}$ (for the drive power $P_{\mathrm{w}} \approx 80 \mathrm{~mW}$ with the cavity resonance $\omega_{c} / 2 \pi=10 \mathrm{GHz},\left|\Delta_{m}\right|,\left|\delta_{c}\right| \sim$ $\omega_{b}$, and $g_{b m} / 2 \pi \approx 1 \mathrm{~Hz}$ ), and the temperature $T=100 \mathrm{mK}$ (i.e., $\bar{n}_{b}^{\text {th }} \approx 200$ ). The local phase $\theta$ in the time-continuous measurement is chosen such that the steering is maximized. It is shown from Figs. 2(a)-2(c) that without the measurement, the weak bipartite steering is achievable and they occur respectively in different parameter regions, but the genuine tripartite is unattainable $\left(S_{c m b}>1 / 4\right)$. This weak bipartite steering originates from the interaction between the magnon and phononic modes in Eq. (3). We note that merely with the interaction in Eq. (3), the genuine tripartite entanglement can be achieved among the cavity, magnon, and phononic modes [16], because entanglement just embodies weaker quantum correlations, compared to quantum steering. However, when the continuous measurement is present, it can be seen from Figs. 2(e)-2(g) that the bipartite steering is enhanced considerably in both regimes of blue $\left(\Delta_{m}<0\right)$ and red $\left(\Delta_{m}>0\right)$ detunings, leading to strong genuine tripartite steering existing prominently in the blue-detuned regime. One can have, for instance, the tripartite steering quantity $S_{c m b} \approx 0.06$ at the detunings $\Delta_{m}=-\omega_{m}$ and $\delta_{c}=-1.8 \omega_{m}$. We can find that the bipartite and genuine tripartite steering in the blue-detuned regime are stronger than those in the red-detuned regime and 

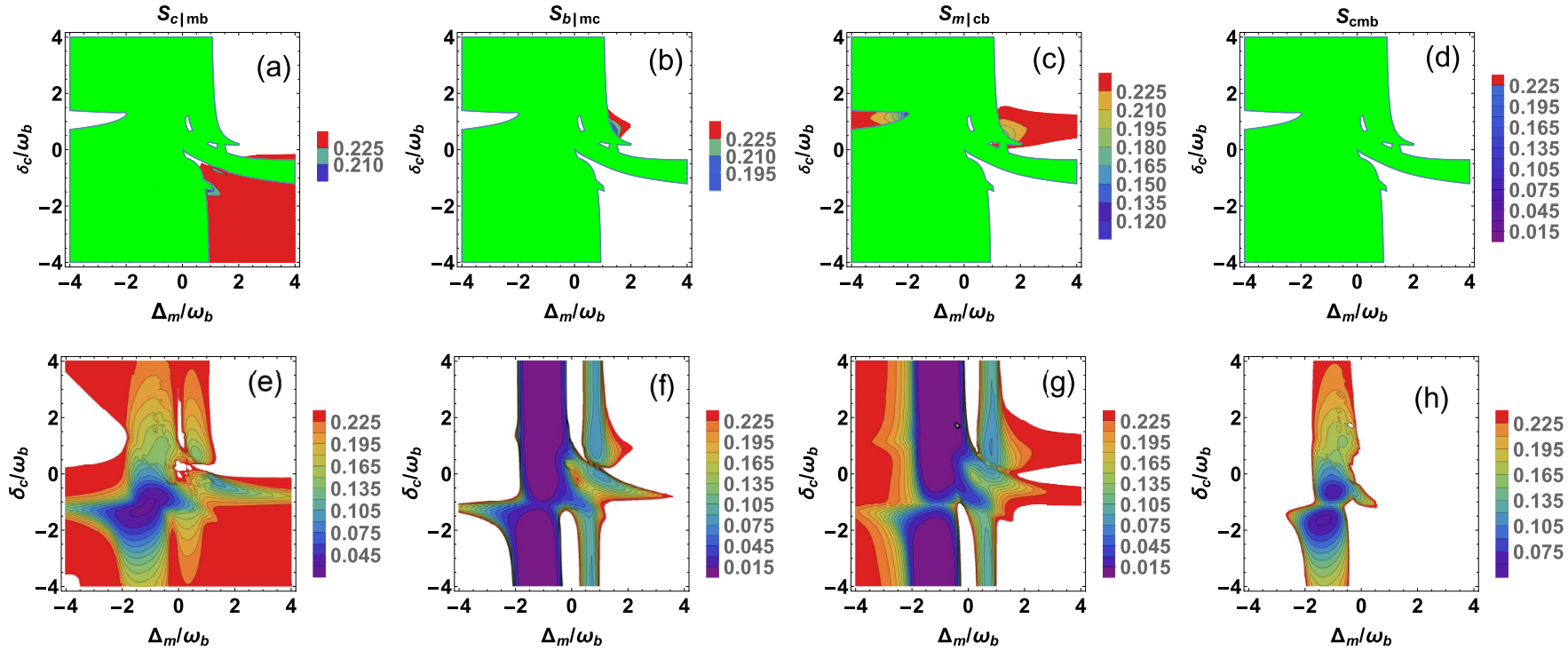

FIG. 2. The dependencies of the bipartite steering $S_{i \mid j k}\left[S_{c \mid m b}\right.$ in (a) and (e), $S_{b \mid m c}$ in (b) and (f), and $S_{m \mid c b}$ in (c) and (g)] and genuine tripartite steering $S_{b m c}$ [in (d) and (h)] on the detunings $\Delta_{m}$ and $\delta_{c}$. In the upper plots, the continuous measurement is absent $(\eta=0)$ and the green shaded areas denote the instability regimes. In the lower plots, the measurement is present with $\eta=1$. The other parameters are $\omega_{b} / 2 \pi=10 \mathrm{MHz}, g_{c m}=G_{b m}=5 \mathrm{MHz}, \kappa_{a} / 2 \pi=3 \mathrm{MHz}, \kappa_{m} / 2 \pi=1 \mathrm{MHz}, \kappa_{b} / 2 \pi=300 \mathrm{~Hz}$, and $T=100 \mathrm{mK}$. In all plots, the white areas represent the steering quantities $S_{i \mid j k}>1 / 4$ and $S_{b m c}>1 / 4$.

further they are optimal at the detuning $\Delta_{m}^{\text {opt }}=-\omega_{b}$. Physically, the steering in the present system originates from the magnon-phonon parametric down-conversion interaction (the term $\left.\hat{m} \hat{b}+\hat{m}^{\dagger} \hat{b}^{\dagger}\right)$ which is dominant over the term $\hat{m} \hat{b}^{\dagger}+\hat{m}^{\dagger} \hat{b}$ (merely leading to state exchange and phonon cooling) in the blue-detuned regime and resonant at the optimal detuning $\Delta_{m}^{\mathrm{opt}}$. Without the measurement, the stability condition of the cavity magnomechanical system severely limits the values of the magnon-phonon coupling $g_{b m}$ in the blue-detuned regime, and therefore one can only achieve the steady-state entanglement, as revealed in Ref. [16], and the bipartite steering which
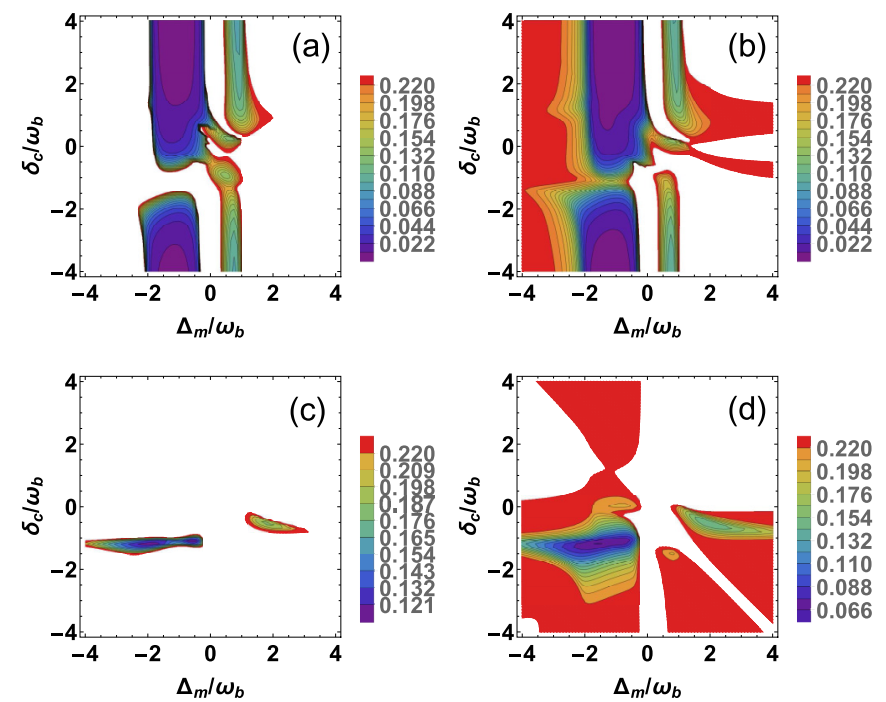

FIG. 3. The dependencies of the two-mode steering $S_{i \mid j}\left[S_{b \mid m}\right.$ in (a), $S_{m \mid b}$ in (b), $S_{c \mid b}$ in (c), and $S_{b \mid c}$ in (d)] on the detunings $\Delta_{m}$ and $\delta_{c}$. The parameters are the same as in Fig. 2 and the white areas represent the quantities $S_{i \mid j}>1 / 4$. are weak such that the genuine tripartite steering is unachievable. When the continuous measurement is present, however, it extends the instability regime to the blue-detuned regime even for larger strengths of the coupling $g_{b m}$, which therefore leads to the strong bipartite and genuine tripartite steering in the blue-detuned regime and moreover they are optimized at the blue detuning $\Delta_{m}^{\mathrm{opt}}$. For the same reason, we see that the bipartite steerings $S_{b \mid c m}$ and $S_{m \mid c b}$ are stronger than the steering $S_{c \mid b m}$ for the same parameters. The above explanation can also be confirmed by investigating the two-mode magnon-phonon steering $\left(S_{m \mid b}\right.$ and $\left.S_{b \mid m}\right)$ which is directly generated via the parametric down-conversion and plotted in Fig. 3. We see from them that the strong magnon-phonon steering in both directions is present in the blue regime and maximizes at the detuning $\Delta_{m}^{\text {opt }}$. Note that the difference in bipartite steerings $S_{b \mid c m}$ and $S_{m \mid b c}$ in the lower panels in Fig. 2 is due to the different loss rates $\kappa_{b}$ and $\kappa_{m}$. In addition, as shown in Fig. 3, mediated by the magnon mode, the photon-phonon steerings $\left(S_{c \mid b}\right.$ and $\left.S_{b \mid c}\right)$ can also be achieved, although they do not interact to each other directly.
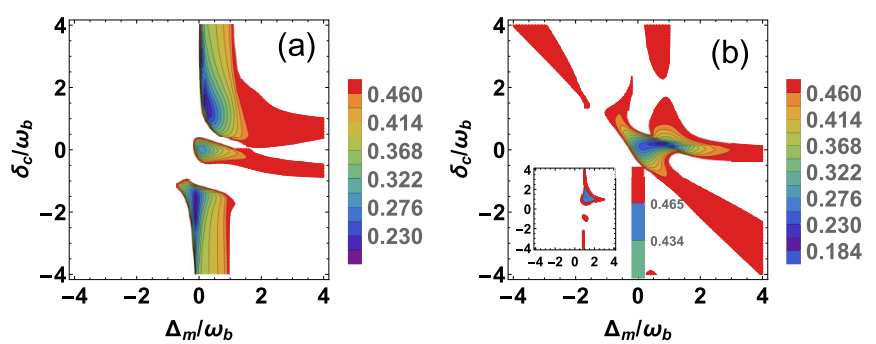

FIG. 4. The dependencies of the quadrature squeezing $V_{m}$ in (a), $V_{c}$ in (b), and $V_{b}$ [the inset in (b)] of the magnons, cavity photons, and phonons on the detunings $\Delta_{m}$ and $\delta_{c}$. The parameters are the same as in Fig. 2 and the white areas represent the variances $V_{m, a, b}>1 / 2$ (no squeezing). 

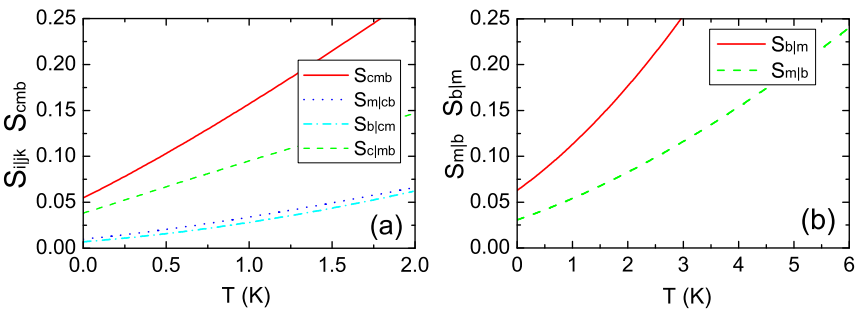

FIG. 5. The dependencies of the bipartite steering $S_{i \mid j k}$ and genuine tripartite $S_{c m b}$ in (a) and the two-mode magnon-phonon steering $S_{b \mid m}$ and $S_{m \mid b}$ in (b) on the temperature for the detuning $\Delta_{m}=-\omega_{b}$ and $\delta_{c}=-1.7 \omega_{b}$. The other parameters are the same as in Fig. 2.

From Fig. 2, we also find that even in the red-detuned regime, the bipartite steering may be improved greatly by the measurement. This is because that the continuous measurement brings about the quadrature squeezing of the cavity, magnon, and phonon modes, which is verified in Fig. 4. We see that the squeezing takes place in the regime of the red detuning. The reduction of the fluctuations leads to the enhancement of the bipartite steering and thus the appearance of the genuine tripartite steering in the red-detuned regime.

In Fig. 5, the effects of thermal fluctuations of the environment on the steering are plotted. We see that the steering is degraded by the thermal phonons but for the given parameters the genuine tripartite steering can exist up to the temperature $T=1.8 \mathrm{~K}$. Also, as shown in the inset, the magnon-phonon steering is much more robust and it can survive even at the temperature $T=6 \mathrm{~K}$. Thus, we see that the bipartite steerings $S_{b \mid c m}$ and $S_{m \mid b c}$ are more robust than the steering $S_{c \mid b m}$ and the all bipartite steering is more robust than the tripartite steering.

\section{DISCUSSION AND CONCLUSION}

After generating the genuine tripartite steering, we should consider how to verify the generated steerable states. To this end, one can employ a weak microwave probing field sent into the cavity to read the state of the magnons. At the same time, by coupling the phonon mode to an optical cavity which is driven by a weak red-detuned light field to induce a beamsplitter-like phonon-photon coupling, the phonon state can be transferred into the optical field [45]. When the dissipation rates of the magnon and phonon modes are much smaller than that of the cavity, by simultaneously homodyning the probing field and the output fields of the microwave and optical cavities and measuring the variances of the generalized quadratures, one can verify the tripartite steering.

It should be emphasized that the present cavity magnomechanical system is merely valid on the condition that the total magnon number $|\langle\hat{M}\rangle|^{2} \ll 2 N$ s to ensure low-lying excitation of the spin ensemble in the YIG sphere, where $N$ is the number of spins and $s=\frac{5}{2}$ is the spin number of the ground-state $\mathrm{Fe}^{3+}$ ion in the sphere. For a $250-\mu$ m-diameter YIG with the spin density $\rho=4.44 \times 10^{27} \mathrm{~m}^{-3}$, we have $N=3.45 \times 10^{16}$, while for $G_{b m} / 2 \pi=5 \mathrm{MHz}$ via choosing the system's parameters in the above, we have $\left|\langle\hat{M}\rangle_{s s}\right|=5 \times 10^{6}$ and thus the condition $\left|\langle\hat{M}\rangle_{s s}\right|^{2}=2.5 \times 10^{13} \ll 2 N s$ is satisfied.

In conclusion, we consider in this paper the generation of genuine photon-magnon-phonon steering in a cavity magnomechanical system via time-continuous homodyne detection on the cavity output. It is found that in the absence of the continuous measurement, only weak bipartite steering among the photons, magnons, and phonons can be obtained. When the measurement is present, the bipartite steering is enhanced considerably and strong genuine photon-magnonphonon steering can be generated in the steady-state regime. The generated tripartite steering is robust against thermal fluctuations. Our scheme provides an approach to observing quantum effects in macroscopic systems, apart from the realistic application to, e.g., one-sided device-independent quantum cryptography and secure quantum teleportation.

\section{ACKNOWLEDGMENT}

This work was supported by the National Natural Science Foundation of China (Grant No. 11674120) and the Fundamental Research Funds for the Central Universities (Grant No. CCNU18TS033).
[1] D. L. Quirion, Y. Tabuchi, A. Gloppe, K. Usami, and Y. Nakamura, Appl. Phys. Express 12, 070101 (2019).

[2] H. Huebl, C. W. Zollitsch, J. Lotze, F. Hocke, M. Greifenstein, A. Marx, R. Gross, and S. T. B. Goennenwein, Phys. Rev. Lett. 111, 127003 (2013).

[3] Y. Tabuchi, S. Ishino, T. Ishikawa, R. Yamazaki, K. Usami, and Y. Nakamura, Phys. Rev. Lett. 113, 083603 (2014).

[4] X. Zhang, N. Zhu, C.-L. Zou, and H. X. Tang, Phys. Rev. Lett. 117, 155301 (2016).

[5] C. Braggio, G. Carugno, M. Guarise, A. Ortolan, and G. Ruoso, Phys. Rev. Lett. 118, 107205 (2017).

[6] D. Zhang, X.-Q. Luo, Y.-P. Wang, T.-F. Li, and J. Q. You, Nat. Commun. 8, 1368 (2017).

[7] Y.-P. Wang, G.-Q. Zhang, D. Zhang, T.-F. Li, C.-M. Hu, and J. Q. You, Phys. Rev. Lett. 120, 057202 (2018).

[8] S. Sharma, Y. M. Blanter, and G. E. W. Bauer, Phys. Rev. Lett. 121, 087205 (2018).
[9] Y. Tabuchi, S. Ishino, A. Noguchi, T. Ishikawa, R. Yamazaki, K. Usami, and Y. Nakamura, Science 349, 405 (2015).

[10] D. Lachance-Quirion, Y. Tabuchi, S. Ishino, A. Noguchi, T. Ishikawa, R. Yamazaki, and Y. Nakamura, Sci. Adv. 3, e1603150 (2017).

[11] Y.-P. Wang, G.-Q. Zhang, D. Xu, T.-F. Li, S.-Y. Zhu, J. S. Tsai, and J. Q. You, arXiv:1903.12498.

[12] X. Zhang, C.-L. Zou, L. Jiang, and H. X. Tang, Sci. Adv. 2, e1501286 (2016)

[13] M. Aspelmeyer, T. J. Kippenberg, and F. Marquardt, Rev. Mod. Phys. 86, 1391 (2014); P. Meystre, Ann. Phys. 525, 215 (2013).

[14] Z. Zhang, M. O. Scully, and G. S. Agarwal, Phys. Rev. Research 1, 023021 (2019).

[15] H. Y. Yuan, S. Zheng, Z. Ficek, Q. Y. He, and M.-H. Yung, arXiv:1903.02484.

[16] J. Li, S.-Y. Zhu, and G. S. Agarwal, Phys. Rev. Lett. 121, 203601 (2018). 
[17] J. Li, S.-Y. Zhu, and G. S. Agarwal, Phys. Rev. A 99, 021801(R) (2019).

[18] J. Li and S.-Y. Zhu, New J. Phys. 21, 085001 (2019).

[19] Z.-X. Liu, H. Xiong, and Y. Wu, arXiv:1910.03738.

[20] E. Schrödinger, Proc. Cambridge Philos. Soc. 31, 553 (1935).

[21] M. D. Reid, P. D. Drummond, W. P. Bowen, E. G. Cavalcanti, P. K. Lam, H. A. Bachor, U. L. Andersen, and G. Leuchs, Rev. Mod. Phys. 81, 1727 (2009).

[22] A. Einstein, B. Podolsky, and N. Rosen, Phys. Rev. 47, 777 (1935).

[23] J. S. Bell, Physics 1, 195 (1964).

[24] H. M. Wiseman, S. J. Jones, and A. C. Doherty, Phys. Rev. Lett. 98, 140402 (2007).

[25] S. J. Jones, H. M. Wiseman, and A. C. Doherty, Phys. Rev. A 76, 052116 (2007).

[26] V. Händchen, T. Eberle, S. Steinlechner, A. Samblowski, T. Franz, R. F. Werner, and R. Schnabel, Nat. Photon. 6, 596 (2012).

[27] S. Wollmann, N. Walk, A. J. Bennet, H. M. Wiseman, and G. J. Pryde, Phys. Rev. Lett. 116, 160403 (2016).

[28] K. Sun, X. J. Ye, J. S. Xu, X. Y. Xu, J. S. Tang, Y. C. Wu, J. L. Chen, C. F. Li, and G. C. Guo, Phys. Rev. Lett. 116, 160404 (2016).

[29] C. Branciard, E. G. Cavalcanti, S. P. Walborn, V. Scarani, and H. M. Wiseman, Phys. Rev. A 85, 010301(R) (2012).

[30] N. Walk, S. Hosseini, J. Geng, O. Thearle, J. Y. Haw, S. Armstrong, S. M. Assad, J. Janousek, T. C. Ralph, T. Symul, H. M. Wiseman, and P. K. Lam, Optica 3, 634 (2016).

[31] M. Piani and J. Watrous, Phys. Rev. Lett. 114, 060404 (2015).
[32] Q. He, L. Rosales-Zárate, G. Adesso, and M. D. Reid, Phys. Rev. Lett. 115, 180502 (2015).

[33] D. Cavalcanti, P. Skrzypczyk, G. H. Aguilar, R. V. Nery, P. H. Souto Ribeiro, and S. P. Walborn, Nat. Commun. 6, 7941 (2015).

[34] S. Armstrong, M. Wang, R. Y. Teh, Q. Gong, Q. He, J. Janousek, H.-A. Bachor, M. D. Reid, and P. K. Lam, Nat. Phys. 11, 167 (2015).

[35] S. Kocsis, M. J. W. Hall, A. J. Bennet, D. J. Saunders, and G. J. Pryde, Nat. Commun. 6, 5886 (2015).

[36] X. Deng, Y. Xiang, C. Tian, G. Adesso, Q. He, Q. Gong, X. Su, C. Xie, and K. Peng, Phys. Rev. Lett. 118, 230501 (2017).

[37] C. Schäfermeier, H. Kerdoncuff, U. B. Hoff, H. Fu, A. Huck, J. Bilek, G. I. Harris, W. P. Bowen, T. Gehring, and U. L. Andersen, Nat. Commun. 7, 13628 (2016).

[38] M. Rossi, N. Kralj, S. Zippilli, R. Natali, A. Borrielli, G. Pandraud, E. Serra, G. Di Giuseppe, and D. Vitali, Phys. Rev. Lett. 119, 123603 (2017).

[39] A. Szorkovszky, G. A. Brawley, A. C. Doherty, and W. P. Bowen, Phys. Rev. Lett. 110, 184301 (2013).

[40] H. M. Wiseman and G. J. Milburn, Quantum Measurement and Control (Cambridge University Press, Cambridge, UK, 2010).

[41] J. Zhang, Y.-X. Liu, R.-B. Wu, K. Jacobs, and F. Nori, Phys. Rep. 679, 1 (2017).

[42] A. A. Clerk, F. Marquardt, and K. Jacobs, New J. Phys. 10, 095010 (2008).

[43] M. D. Reid, Phys. Rev. A 40, 913 (1989).

[44] Q. Y. He and M. D. Reid, Phys. Rev. Lett. 111, 250403 (2013).

[45] D. Vitali, S. Gigan, A. Ferreira, H. R. Böhm, P. Tombesi, A. Guerreiro, V. Vedral, A. Zeilinger, and M. Aspelmeyer, Phys. Rev. Lett. 98, 030405 (2007). 